\title{
Special Issue About "Recent Advances on MOBILe Wireless MiddleWARE, Operating Systems, and Applications"
}

\author{
Jean-Marie Bonnin - Thomas Magedanz
}

Published online: 27 April 2010

(C) Springer Science+Business Media, LLC 2010

The advances in wireless communication technologies and the proliferation of mobile devices have enabled the realization of intelligent or smarter environments for people to communicate with each other, interact with information processing devices, and receive a wide range of mobile wireless services through various types of networks and systems everywhere and anytime. This emerging "Internet of Things" will dramatically modify our lives, allowing progress in various domains, such as health support, ITS (Intelligent Transportation Systems), energy efficiency, and security. A key enabler of this pervasive and ubiquitous connectivity environment is the advancement of software technologies in various communication sectors, ranging from sensor technologies, operating systems, communication middleware to networking protocols and applications.

The international ICST conference series on Mobile Wireless Middleware, Operating Systems, and Applications (MOBILWARE) is dedicated to address these emerging topics and challenges in various mobile wireless software-related areas. The scope of the conference

\footnotetext{
J.-M. Bonnin $(\bowtie)$

Institut Telecom / Telecom Bretagne,

Université européenne de Bretagne,

Rennes, France

e-mail: jm.bonnin@telecom-bretagne.eu

T. Magedanz

Technische Universität Berlin,

Berlin, Germany

e-mail: thomas.magedanz@tu-berlin.de
}

includes the design, implementation, deployment, and evaluation of middleware, operating systems, and applications for computing and communications in mobile wireless systems. This special issue features extensions of five of the best papers of the MOBILWARE 2009 conference, which took place in Berlin, Germany in April 2009 and also illustrates accurately the scope of the conference.

The incredible development of mobile devices leads most of us to have a large processing capacity in the pocket. Nevertheless some applications still require too much processing to be easily implementable in smart phones. In the first paper, André C. Santos et al. study the possibility to implement an autonomous navigation algorithm on smart-phones using the standard Java machine (J2ME). This algorithm controls a robot and relies on image processing and path planning which are quiet demanding.

Even if it is possible to perform demanding tasks on a smart phone, it is reasonable to rely on the ubiquitous Internet connectivity to use servers in the cloud to alleviate processing and battery constraints. The behavior of the network could be adapted to the context in which the mobile terminal operates to be more efficient and to provide a better service to the customer. Josephine Antoniou et al. present in the second paper a multicast session management framework which is able to create and evolve session depending on the terminal context.

Simplifying the development of new applications and reducing the time to market for new applications and services is also a challenge that has to be tackled. Florian 
Deinert and al. propose in the third paper to use the widget concept to develop rapidly IMS client applications using IMS features provided by operators. The architecture consists in a widget engine (available on Android) and a collection of widgets (web application) using the feature it exposes.

Besides mobile terminals using mobile operator infrastructures to communicate with the Internet and between them, devices can organize autonomously their interactions to perform various tasks. In the Internet of Things heterogeneous sensor and actuator devices have to collaborate in order to perform various tasks.

For example a European Smart Cities project plans to deploy 25,000 heterogeneous sensors in Santander (Spain). In the 4th article, Sergio Gonzalez proposes a tool to ease the deployment of applications in wireless sensor networks. Mobile agents perform a given task (e.g. collect data) while traveling over the network. As shown in the article, the proposed programmable middleware allows to execute mobile agents efficiently even on tiny devices.

In the last paper, Amir R. Khapour et al. aim at providing an efficient event service over spontaneous networks (ad-hoc networks). The proposed system adopts a cross layer approach to use as efficiently as possible underlying network level mechanisms (Multipoint Relays of OLSR) to provide a secure message delivery service. The designed persistent event service supports mobility and resists to transient disconnection and to network partition.

We want to express our sincere gratitude to all the authors who have submitted their papers to this special issue and to all the reviewers whose accurate work was crucial for the high-quality of the papers.

We hope that all readers will enjoy the reading.

Jean-Marie Bonnin and Thomas Magedanz

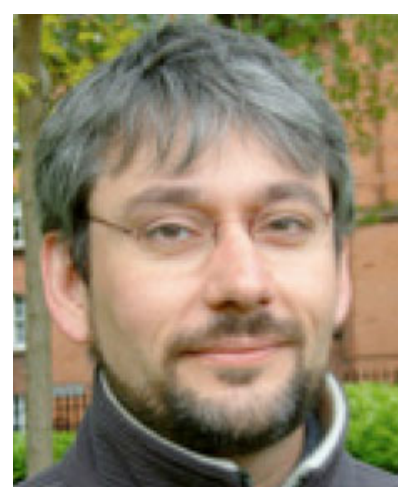

Professor Jean-Marie Bonnin got his $\mathrm{PhD}$ degree in Computer Science at the University of Strasbourg, France in 1998. He has been with Telecom Bretagne since 2001, where he is currently the head of the "Networks, Security and Multimedia" (RSM) department. He also leads the Mobility research team in the RSM dept. His main research interests lie in the convergence between IP networks and mobile telephony networks, and especially in heterogeneous handover issues. Recently, he has been involved in projects dealing with network mobility and its application to ITS (Intelligent Transportation Systems).

$\mathrm{He}$ is involved in several collaborative research projects at the French and European levels and through international academic collaborations (mainly with Asia and North Africa).

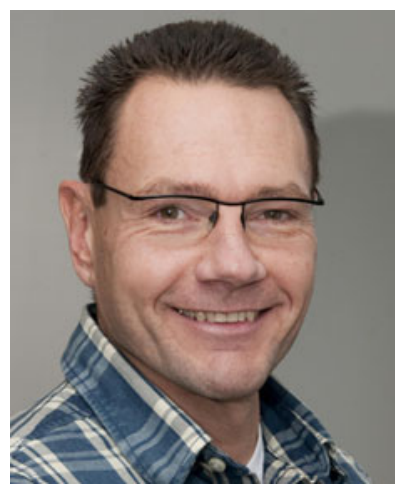

Thomas Magedanz (PhD) is professor in the electrical engineering and computer sciences faculty at the Technische Universität Berlin, Germany. In addition, he is director of the "next generation network infrastructure" division of the Fraunhofer Institute FOKUS, which provides various test beds and tool kits enabling advanced research and education in the context of open service delivery platforms and related middleware. Since more than 20 years Prof. Magedanz is working in the convergence field of fixed and mobile telecommunications, the internet and information technologies. In addition, Prof Magedanz is senior member of the IEEE, and editorial board member of several international journals. In 2007, Prof. Magedanz joined the European FIRE (Future Internet Research and Experimental Facilities) Expert Group. 